\title{
Les nœuds et les liens du réseau Internet : approche géographique, économique, et technique
}

\section{Introduction}

Beaucoup estiment encore aujourd'hui qu'Internet est un réseau décentralisé et sans hiérarchie, a-spatial voire déterritorialisé, gratuit ou presque dont le contenu serait partagé par une communauté (l'humanité) au sein de laquelle chacun aurait vocation à être ou devenir producteur. Le rêve de l'Internet gratuit, décentralisateur, universel, aux valeurs démocratiques a laissé place à une réalité bien différente. L'accès à cette technologie n'est pas universel : des lieux sont très bien connectés, d'autres à l'écart. L'Internet n'est pas véritablement un « bien commun » car il demeure contrôlé par les Etats-Unis et quelques grandes entreprises. Ses vertus décentralisatrices n'ont rien d'automatiques et des processus inverses peuvent être observés [Brown, 2002]. Enfin, sa « gratuité » n'est qu'apparente. Il produit de la valeur et fait donc l'objet d'une féroce compétition entre acteurs économiques (propriétaires des infrastructures, fournisseurs de services) ou politiques (agences gouvernementales, acteurs territoriaux). Et paradoxalement, la chute continuelle des prix, voire la prétendue gratuité qui ne touche que certains territoires est le résultat provisoire de cette bataille et ne saurait durer pour les utilisateurs finaux (ménages, collectivités locales, entreprises) qui coproduisent l'Internet mondial. La question du partage de cette valeur nous semble essentielle pour saisir l'articulation entre le développement du réseau et les territoires, les lieux de l'interconnexion qui restent les lieux de forte polarisation et de diffusion de l'innovation.

Notre grille d'analyse privilégie les interactions de plusieurs variables : l'histoire, qui a tracé des chemins et donné des avantages comparatifs souvent décisifs ; l'évolution technologique, qui modifie les possibles et rebat les cartes entre territoires et acteurs ; la culture des développeurs de l'Internet, qui a favorisé dans un premier temps l'interconnexion des réseaux.

Si l'architecture des télécommunications obéit aux règles classiques des réseaux, elle se différencie sur le plan technico-économique. Aussi faut-il explorer un peu plus loin la boîte noire des techniques et les principes de développement géoéconomiques pour comprendre les contraintes de la diffusion de l'accès aux télécommunications à haut débit. Celles-ci s'appuient sur des équipements particuliers du « cyberespace » ${ }^{1}$ (GIX, points de présence, répartiteurs, etc.), dont la localisation détermine les conditions de l'accès et contraint à des localisations « de proximité » (data center, serveur). En quoi ces équipements structurent-ils la forme des réseaux et surtout quelles en sont les contraintes ? Quels éléments, l'étude géographique doit-elle prendre en compte pour expliciter le développement des réseaux à haut débit et les inégalités d'accès entre les territoires?

\section{Méthode et bases de recherche}

Cette analyse s'appuie essentiellement sur des travaux scientifiques existants dans le domaine de la géographie, de l'économie, des sciences de gestion et des sciences techniques. En géographie, les productions anglosaxonnes sont de loin les plus nombreuses : les chercheurs américains comme E. Malecki, M. O’Kelly, T. Grubesic ont été les premiers à s'interroger sur la géographie du réseau. Quelques pistes ont été tracées en Europe avec les Anglais M. Dodge ${ }^{2}$, Gillepsie, J. Rutherford, ou encore G. Dupuy en France.

De nombreux rapports, issus de différents organismes de consultant, des études de cas parues dans la presse spécialisée ou produits par des acteurs de l'Internet ont été pris en compte. La principale source reste Telegeography qui fournit de nombreuses données quantitatives (trafic) et qualitatives (cartes). Les sites des opérateurs offrent également des informations intéressantes, concernant leur stratégie commerciale et la localisation de leur réseau. Ces matériaux, nombreux, riches et essentiellement publiés en anglais, appartiennent à différents champs disciplinaires et sont difficiles à évaluer, d'autant que la technologie et ses contextes d'utilisation évoluent rapidement, et surtout que les interprétations heuristiques et les intérêts des acteurs divergent grandement.

Beaucoup de temps a été consacré par les auteurs pour comprendre les enjeux technico-géographiques de la topologie du réseau et pour faire lire et vérifier les travaux par plusieurs acteurs issus du monde de la technique.

\footnotetext{
${ }^{1}$ Par cyberespace, nous parlons ici de cyberplace ou de cyberlieu, un des quatre espaces de la géographie virtuelle créé par des ordinateurs et des communications selon Batty (1997). Le cyberplace se compose de tous les fils qui constituent les réseaux et de leurs infrastructures, y compris les réseaux sans fil.

${ }^{2}$ S'il existe peu de cartes des routes de l'Internet, le géographe anglais Martin Dodge est l'un des seuls à avoir recueilli de nombreuses données pour construire son Atlas de l'Internet.
} 
La première partie se consacre à la géographie de l'Internet en analysant successivement : les logiques de déploiement des routes et des flux, qui créent des liens privilégiés entre pays développés ; les nœuds d'interconnexion des réseaux qui polarisent les dynamiques et complexifient les rapports territoriaux; et enfin, la localisation des contenus qui représentent une création potentielle de valeur. L'analyse s'accompagne de représentations géographiques de l'Internet. La seconde partie vise à comprendre les principes qui régissent « l'écosystème d'Internet » en s'appuyant sur les mutations du marché, mais aussi sur les stratégies d'acteurs et la question de la valeur. L'analyse montre que même si l'évolution d'Internet a mené à d'importantes restructurations sectorielles et à la disparition de certains acteurs, il n'en demeure pas moins que le marché reste concentré autour de quelques opérateurs, surtout américains. En conclusion quelques pistes de réflexion seront proposées pour comprendre le degré de stabilité de l'écosystème Internet et ses facteurs éventuels d'entropie.

\section{Internet en cartes}

\section{Où sont les tuyaux ?}

Si Internet est bien un phénomène multidimensionnel, son existence repose sur une infrastructure mesurable à la fois par des réseaux physiques et des équipements qui permettent l'interconnexion des communications électroniques. Il a été conçu comme un réseau maillé, dont la redondance sert à résister aux pannes et aux avaries. Or, sa structure actuelle ressemble bien plus, au niveau mondial, à une étoile centrée sur les États-Unis.

\section{Les routes de l'Internet}

Le poids de l'histoire et du chemin tracé par la technique de transmission de la voix (téléphonie) est un premier facteur explicatif. Les marchés qui généraient le plus de valeur étant les deux marchés anglophones des EtatsUnis et de la Grande-Bretagne, les routes transatlantiques se sont développées les premières. Ainsi, le développement des dorsales de l'Internet (backbones) a suivi les câbles de la téléphonie sur longue distance. Face aux premiers opérateurs, apparus en 1996-98, contrôlant les grandes dorsales, les nouveaux arrivants se sont trouvés très vite dans l'obligation de s'interconnecter avec eux pour atteindre les utilisateurs. Derrière le terme opérateurs, il faut entendre l'ensemble des propriétaires d'infrastructures longue distance (IBP, Internet Backbone Providers) ainsi que les FAI, fournisseurs d'accès à Internet. L'attraction des routes les plus profitables a donc renforcé les positions des premiers opérateurs : ceux que Malecki (2003) appelle les « vieux garçons des réseaux »: Cable \& Wireless, GTE Internetworking (aujourd'hui, Genuity), PSInet, Sprint et UUNET (aujourd'hui MCI/ Worldcom). Des trois ensembles de la «triade », l'Asie pacifique demeure en retrait, mais c'est surtout vers les pays du Sud (Afrique, Asie du Sud Est et Amérique Latine) que les flux sont les plus faibles.

\section{Le trafic interrégional en 2004}

La domination des liens transatlantiques est écrasante : $66 \%$ des flux globaux. Ces flux ont augmenté de $250 \%$ entre 2002 et 2004 mais ont connu un recul relatif $(71 \%$ en 2002) car la croissance des volumes entre l'Amérique du Nord et l'Asie ou l'Amérique latine a été plus rapide. Pour la même période, l'Afrique a vu le volume de ses flux tripler avec l'Europe et doubler avec l'Amérique du Nord. Dans ce contexte géopolitique, les pays du Nord et surtout les Etats-Unis occupent une place centrale: 98,7 \% des flux interrégionaux passent par le continent nord-américain. 


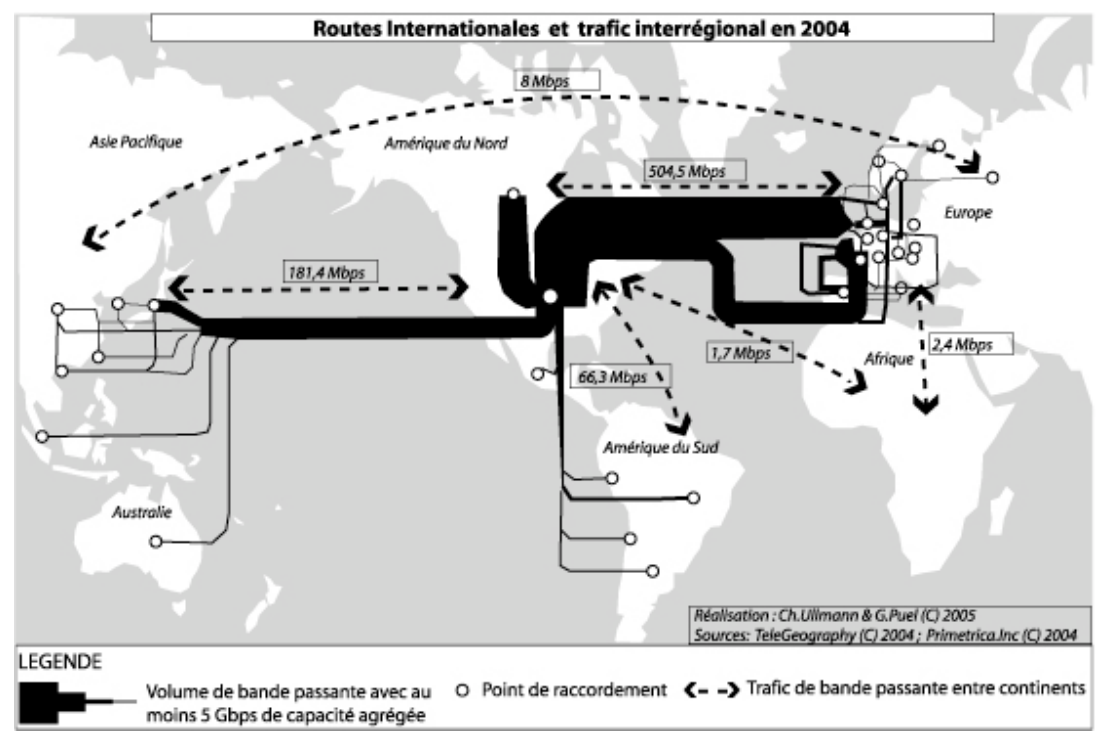

Fig. 1 / Routes internationales et trafic interrégional en 2004

La distribution de la bande passante montre l'importance des routes transocéaniques pour les grands opérateurs. $85 \%$ du trafic concerne Internet, le reste rassemble les échanges des réseaux privés et les communications téléphoniques.

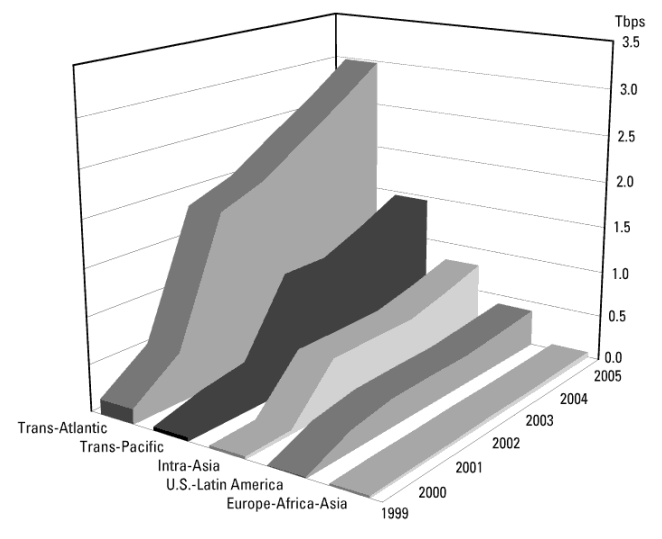

Fig. 2 / Evolution de la capacité utilisée des câbles sous-marins : 1999-2006

Telegeography estime qu'en 2004 seulement 25\% de la capacité des câbles transatlantiques est utilisée. Toutefois ces chiffres ne représentent qu'une moyenne ne tenant pas compte des pics d'utilisation.

Ce graphique témoigne de l'évolution des flux entre les espaces régionaux de 1999 à 2005. La route transatlantique domine toujours et l'encéphalogramme demeure désespérément plat entre l'Europe et l'Afrique ${ }^{3}$ ou l'Asie. Après une phase de décollages des flux, la croissance du trafic fléchit en 2001 (éclatement de la bulle Internet). La période 2004-2005 annonce une reprise, témoignant du regain de confiance des usagers et des investisseurs, et anticipant l'explosion du trafic due aux progrès de l'accès au réseau et aux nouveaux usages (voix sur IP par exemple).

\footnotetext{
${ }^{3}$ Bien que le volume des flux soit en forte croissance sur la période récente, il demeure peu significatif à l'échelle mondiale $(0.005 \%)$ et difficilement cartographiable.
} 


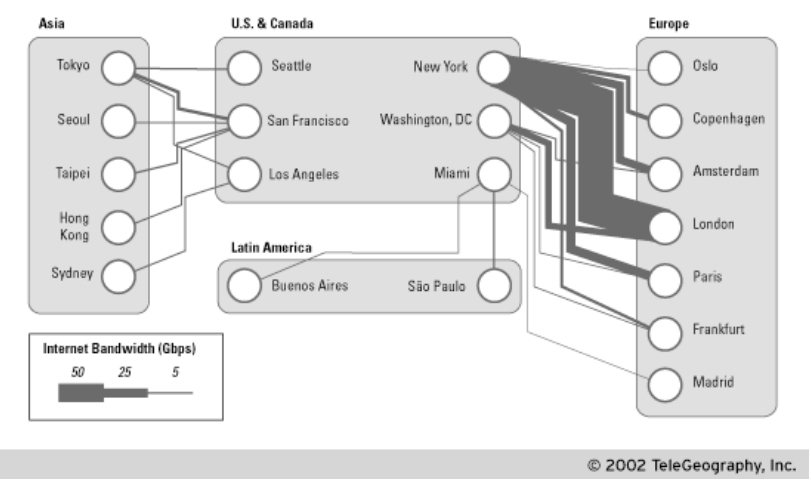

Fig. 3 / Principaux nœuds d'échanges internationaux, en volume de bande passante

\section{Où sont les nouds?}

Internet, réseau des réseaux, permet grâce à un protocole (IP) d'interconnecter des milliers de réseaux d'essence différente, opération transparente pour l'internaute. Les opérateurs doivent donc s'échanger leurs "paquets» d'informations en un lieu, le nœud d'échange, GIX (Global Internet eXchange) ou NAP (Network Access Point), où sont implantés les équipements électroniques nécessaires. La structure d'Internet permet ainsi aux plus petits réseaux d'être interconnectés avec les grands réseaux de backbones, eux-mêmes interconnectés.

\section{Les logiques de déploiement des nœuds (GIX)}

La multiplication ou non du nombre de nœuds est une question encore difficile à résoudre. Deux logiques technico-économiques s'opposent.

L'une conduit à multiplier les GIX, mettant en avant certains avantages techniques : des circuits et des temps de réaction plus courts, un désengorgement des grandes artères, une meilleure efficacité pour les usages liés au haut débit. L'adoption de ce modèle, adossé à des GIX locaux, améliorerait la performance économique et serait le garant du développement territorial. Derrière ces arguments se retrouvent le mythe de l'Internet décentralisé et le souhait, partagé par tous les utilisateurs et les politiques publiques, de voir les acteurs économiques pratiquer l'interconnexion et la concurrence les plus larges possibles.

La deuxième logique privilégie un nombre restreint de GIX, mettant ${ }^{1}$ en avant la complexité technique du routage qui nécessite de réduire le nombre d'acteurs pour diminuer les incidents de réseau. « Le routage est une tâche complexe (elle dépend du nombre de FAI participant à l'échange) et très sensible (une erreur locale peut avoir des répercussions majeures dans tout l'Internet) qui réclame à la fois des personnels très compétents et disponibles et des cadres organisationnels clairs. Les mécanismes évoqués précédemment permettent un changement d'échelle contrôlé, changement que réclame l'explosion du nombre d'acteurs. ${ }^{4} »$

\footnotetext{
${ }^{4}$ http://mission-dti.inria.fr/, consulté le 11/12/2004.
} 


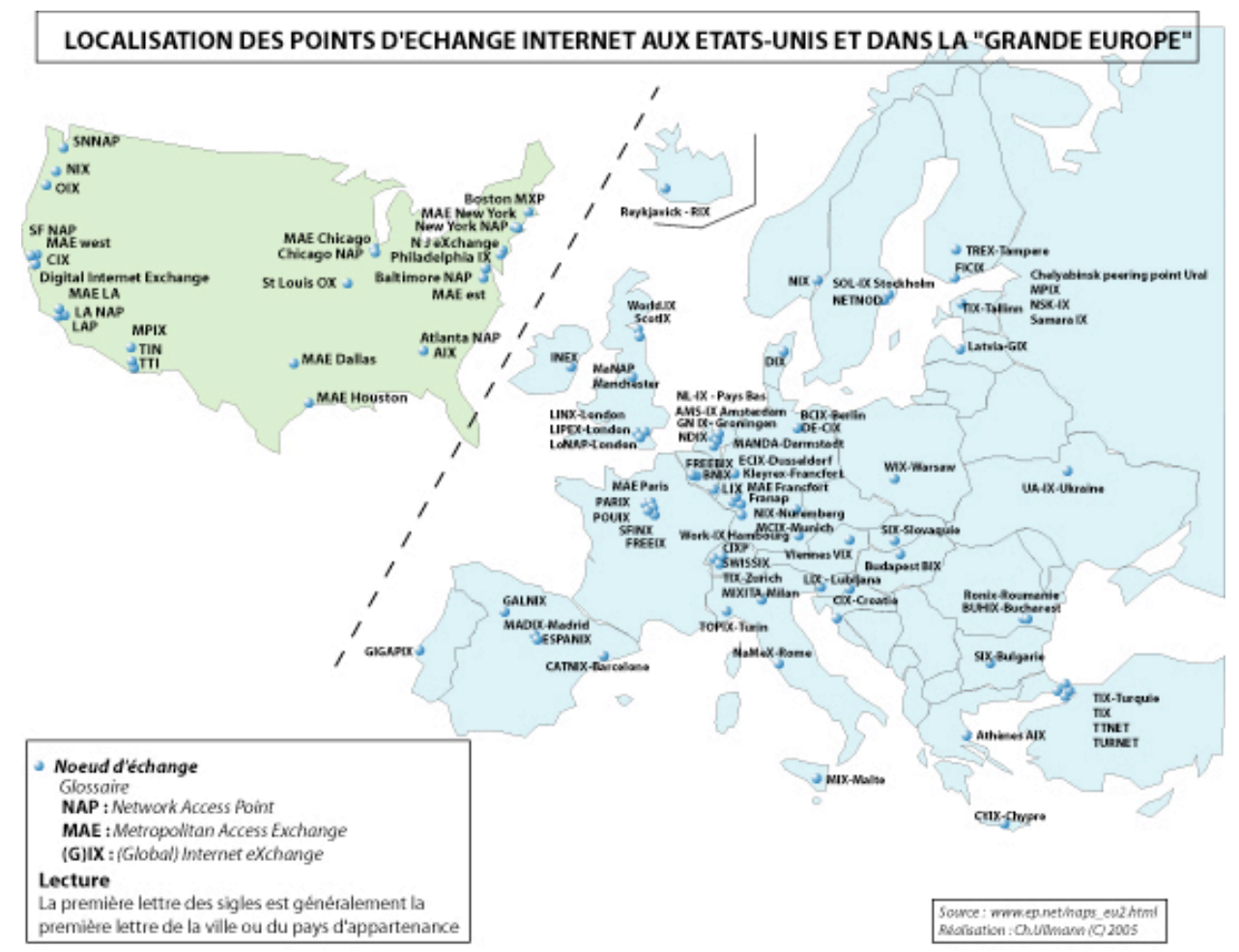

Fig. 4 / Localisation des points d'échange internet aux Etats-Unis et dans la « Grande Europe »

\section{Les modèles de l'interconnexion}

L'interconnexion suit deux modèles économiques (le peering et le transit) selon les tailles respectives des acteurs. Les plus grands opérateurs ( les tier one) déploient de très nombreux points d'interconnexion privés deux à deux et pratiquent le peering privé, sans échange monétaire ; ils participent aux GIX publics essentiellement pour revendre (transit) de la capacité et des services aux opérateurs de moindre importance. Les GIX sont les points d'interconnexion des réseaux, mais tous les opérateurs présents en un GIX ne sont pas nécessairement interconnectés.

Dans les GIX, la réciprocité est basée sur un système de plus en plus hiérarchisé, emboîtant les échelles géographiques. Un premier niveau permet aux plus gros opérateurs la pratique du peering mais aussi le regroupement dans ces quelques rares pôles des compétences techniques les plus pointues. Un deuxième niveau regroupe les opérateurs régionaux qui peerent entre eux et achètent de la bande passante (transit) aux opérateurs du premier niveau ; ils concentrent des compétences techniques de niveau moindre. Enfin un troisième niveau rassemble des FAI, de taille plus modeste, qui achètent ou louent de la bande passante et des compétences techniques aux plus gros opérateurs.

Si des éléments techniques (qualité de service), politiques ou économiques (business model) participent au choix des arrangements de peering, ils ne constituent pas les seuls critères. W.B Norton (1999) montre que les alliances stratégiques entre opérateurs sont souvent plus importantes que toutes les autres conditions. Par exemple, France Télécom évite le peering avec certains de ses concurrents directs comme Cegetel, même s'ils sont présents sur des GIX communs. Ils préfèrent s'interconnecter dans un GIX privé situé aux Etats-Unis. La politique de peering de France Télécom impose que les candidats possèdent des nœuds de réseaux ainsi que des clients IP dans les cinq communautés urbaines de Paris, Lyon, Toulouse, Marseille et Lille. De même, les tier one privilégient les accords de transit avec les petits opérateurs afin de conserver une position dominante. «Telephone companies, who are no strangers to the strategic use of interconnection, now own the major internet backbones. The Internet has become increasingly commercial and profit-oriented, which explains the restrictions on free peering imposed by IBPs and the cable companies resistance to open access » (Mah 2003). 


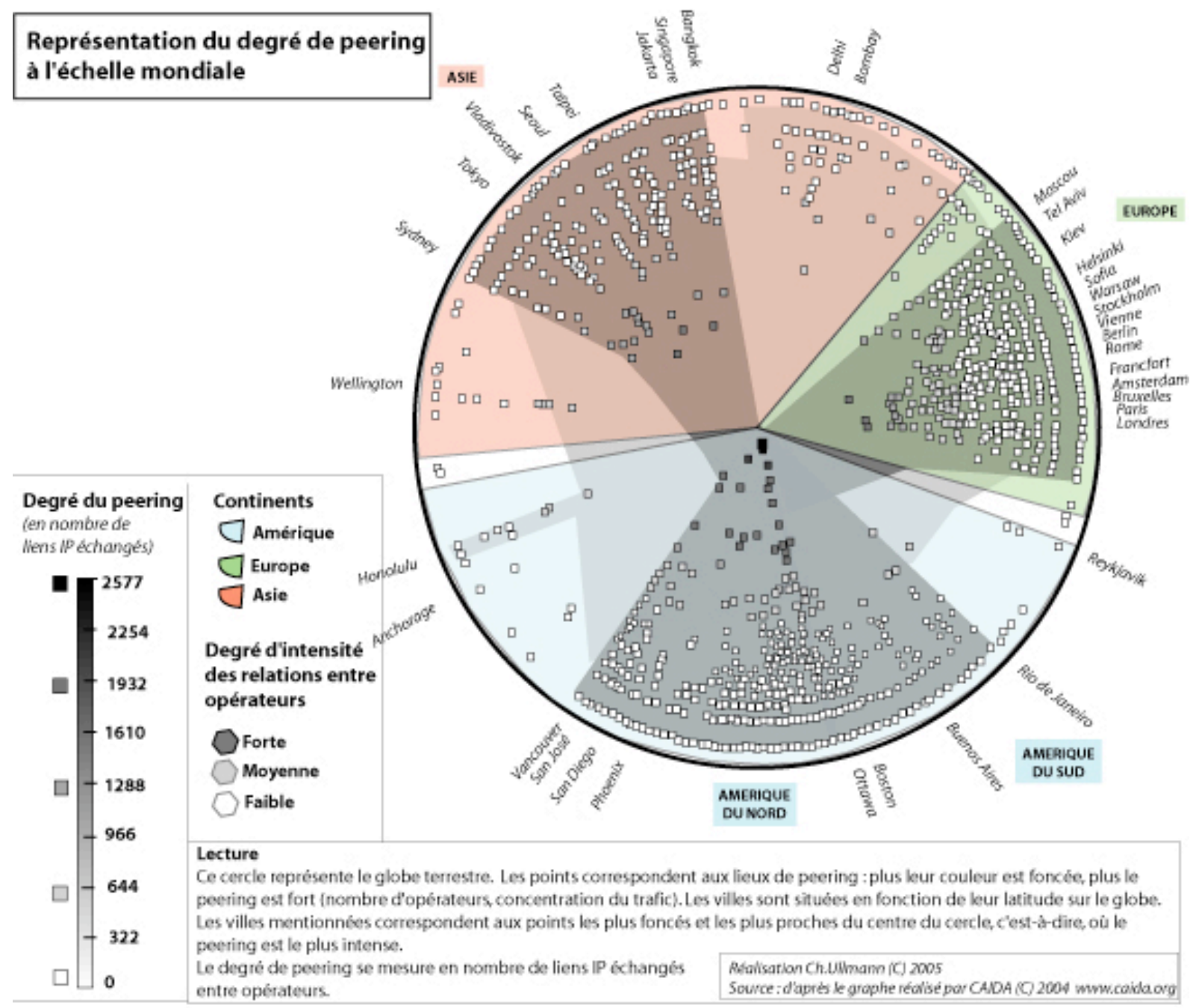

Fig. 5/ Intensité des échanges entre opérateurs de télécommunications

\section{La connectivité internationale des villes}

La diffusion spatiale des points d'échange est un repère barométrique de la compétition autour des infrastructures Internet. Elle est liée à la concentration des activités et des compétences. Ainsi se dessine une industrie de l'interconnexion qui s'appuie sur des équipements techniques mais également sur des besoins réguliers de maintenance dans les salles spécialisées. Plus un nœud d'interconnexion est fréquenté, plus il devient un lieu stratégique.

En Europe, si chaque pays dispose aujourd'hui de son GIX, une hiérarchie se dessine. Le rôle des villes en tant que « nœuds » est d'autant plus fondamental que le réseau Internet représente une nouvelle forme de l'infrastructure urbaine (Graham \& Marvin, 20001). En 2000, les villes européennes les plus connectées étaient Londres (20 nœuds), Amsterdam (19 nœuds), puis Francfort, Hambourg et Paris (18 nœuds). 


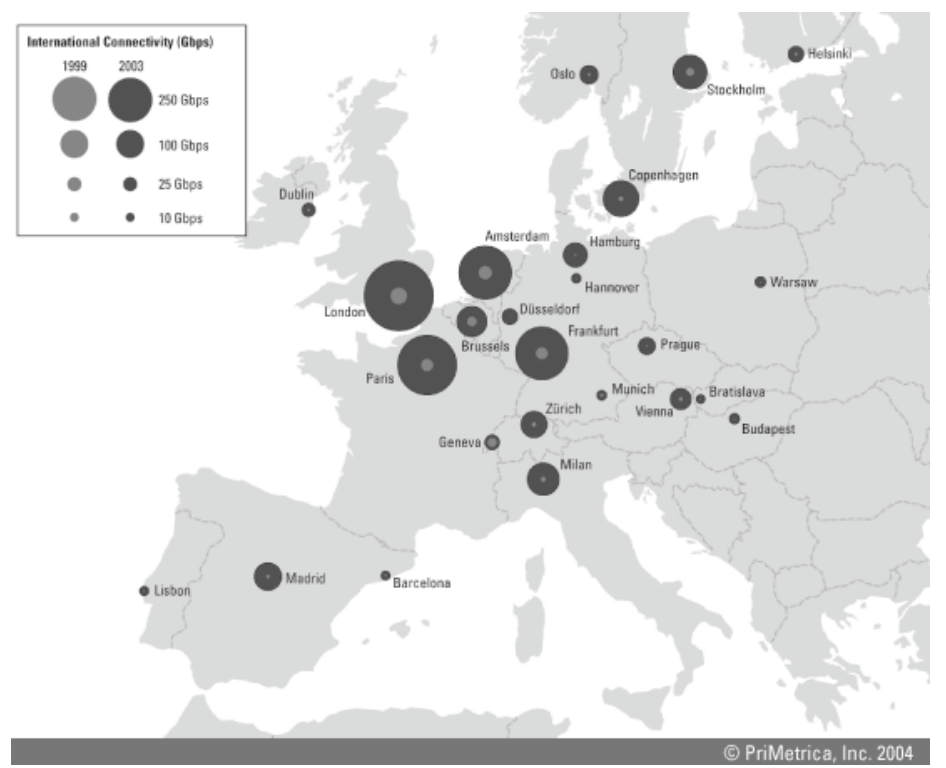

Fig. 6/Connectivité des grandes villes européennes vers les réseaux longue distance

La demande de bande passante croît fortement malgré l'éclatement de la bulle. En 1999, Londres consommait 34 Gbits puis 620 Gbits en 2003. L'augmentation de la capacité des débits ne s'est pas faite uniformément dans l'espace : ce sont essentiellement les très grands centres urbains qui en ont bénéficié. Mais les villes subissent ce que Wheeler et O'Kelly (1999) appellent « the end of the track phenomenon », c'est-à-dire que l'accessibilité aux grandes artères de télécommunications diminue en fonction de l'éloignement des villes du cœur de l'Internet. Il précise que si l'on détruit un nœud d'interconnexion principal, on détruit automatiquement tous les liens qui y sont raccrochés, provocant d'importants effets sur la structure globale de l'Internet. Les premiers lieux touchés seraient les petites et moyennes villes, car les grandes métropoles bénéficiant de leur situation de $h u b$, peuvent mobiliser les autres réseaux disponibles. Internet est bien un réseau fortement hiérarchisé et dépendant de la géographie des lieux.

Les villes concentrent l'essentiel des capacités technologiques, mais en réalité, à peine 4 à $5 \%$ de la bande passante mondiale sont véritablement utilisés. L'exemple type est celui de la petite ville de 26.000 habitants d'Helena au Montana qui a accès à un potentiel de 113 Tbits de bande passante, soit quatre fois plus que la totalité consommée aux Etats-Unis en juin 2003 ! « Le surplus de bande passante et d'infrastructures est notre pire problème dans le Montana » témoigne G. Fiess, le directeur général de l'Association des Télécommunications du Montana. « Il existe des aberrations dans la situation technologique de notre région. Par exemple, bien qu'il y ait plus de $9.000 \mathrm{Km}$ de lignes optiques et près de 90 lieux de téléconférences, la plupart des services ne sont pas disponibles sur les lieux de vie des habitants $»^{5}$.

\section{Où sont les contenus ?}

La répartition de la bande passante se double de la question de l'accès (quels coûts, pour quels usagers, pour quels contenus, etc.). La localisation des contenus (site ou page web) a été mesurée de deux manières : soit par le nombre de sites identifiés selon la localisation de l'enregistrement des noms de domaine (Zook, 2000) ou de leur territoire d'appartenance (Duféal, 2004) ; soit par la localisation des équipements matériels qui abritent par exemple les serveurs (data centers).

\section{Les sites web}

Zook localise les noms de domaines en CONE (EDU/ORG/NET/COM/INT) et CC (Country Code) selon leur adresse d'enregistrement. Cependant, il faut savoir qu'il n'y a aucun lien entre un nom de domaine en CC et sa localisation : ainsi un site d'entreprise allemand en « .de » peut très bien se trouver aux Etats-Unis. Zook évalue la fiabilité de sa méthodologie à $84 \%$.

Cette carte confirme la hiérarchie urbaine européenne et révèle la situation privilégiée de Londres. Certains pays comme la France, le Royaume-Uni et les Pays-Bas possèdent des pôles d'agrégation des noms de domaines sur les capitales nationales; d'autres comme l'Allemagne montre une distribution décentralisée qui suit la hiérarchie urbaine fédérale.

\footnotetext{
${ }^{5}$ http://www.mtinbusiness.com/inbusinessspring03/bus06.html, in Western Montana in Business, 2003, consulté le 7/12/2004.
} 


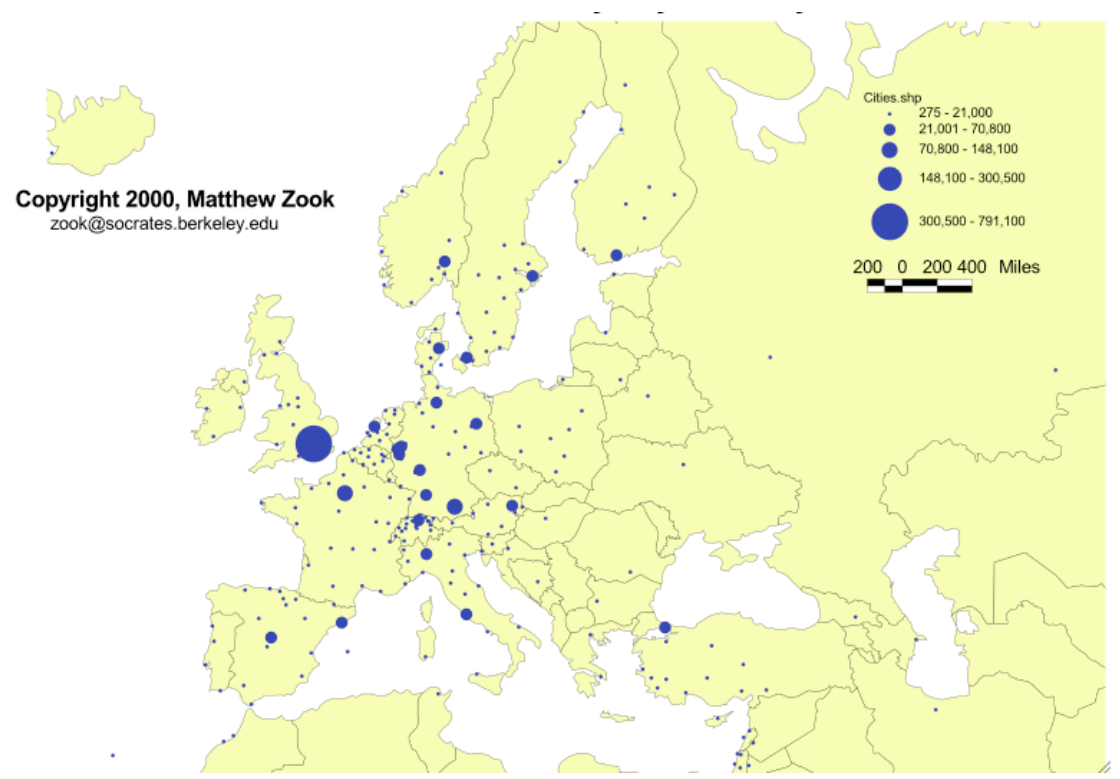

Fig. 7/ Densité des sites web en Europe

Recensant le nombre de sites produits par les acteurs locaux (entreprises, administrations, etc.), Duféal montre qu'ils représentent de façon plus ou moins fine la territorialisation des activités économiques. La densité des sites est liée à la densité de la population et à l'intensité des activités économiques.

\section{Les équipements stockant les contenus : serveurs, data centers}

Le contenu de l'Internet, essentiellement localisé aux Etats-Unis, exerce un attrait immense pour les internautes du monde entier alors que l'attrait pour des contenus ou services «locaux» et territorialisés reste à prouver. Il en découle une asymétrie dans les échanges qui pénalise les opérateurs européens et les conduit à accroître leur capacité sur les routes transocéaniques.

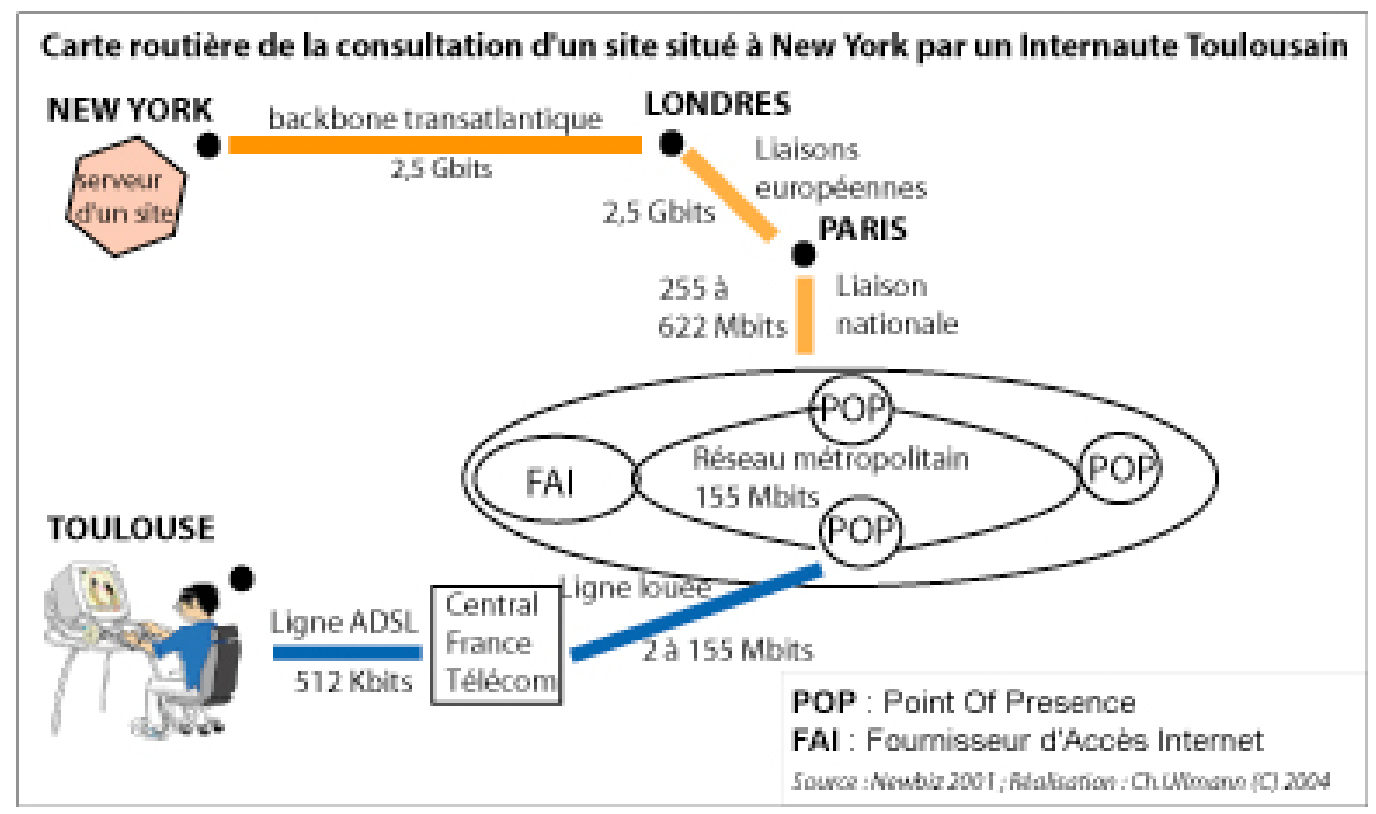

Fig. 8/ Le trajet d'une requête

En 2001, les 50 pays d'Afrique subsaharienne (hors Afrique du Sud) ne comptaient pas plus de sites que la seule Lituanie ! En outre, les sites africains les plus intéressants se sont «délocalisés» en Europe et en Amérique du Nord à cause de la meilleure connexité Internet (J-L. Fullsack, 2002). De même, le transit des communications téléphoniques internationales entre Etats africains passe presque exclusivement par des plateformes de commutation européennes. 
Il peut s'avérer plus stratégique et profitable pour une entreprise européenne de louer un serveur pour l'hébergement aux Etats-Unis qu'en Europe. Pour atteindre ses clients en Asie ou en Europe, les coûts sont moindres et les temps de consultation des pages plus rapides ; de facto le site aura plus de chance d'être visité par les internautes américains qui sont les plus nombreux. Cukier (1999) estime que l'hébergement d'un site non américain aux Etats-Unis améliore son audience et son fonctionnement, mais souligne tout de même la difficulté à trouver des données fiables vérifiant son hypothèse.

Ainsi, les opérateurs préfèrent localiser les data centers dans les métropoles qui offrent la meilleure connectivité possible avec les réseaux longue distance. De même, les compétences techniques nécessaires à la maintenance du système sont rares et localisées à proximité des grands noeuds internationaux. Enfin, leur marché vise les multinationales qui cherchent une audience mondiale et une qualité de service maximale. A titre d'exemple, les chiffres du tableau ci-dessous correspondent aux connexions de 155 Mbits en longue distance vers les réseaux métropolitains assurés par les opérateurs. Le réseau de MCI interconnecte 28 de ces réseaux longue distance sur la ville de New York, 10 sur Miami ou Londres, et seulement 5 sur Paris.

\begin{tabular}{|c|c|c|c|c|c|c|c|c|c|}
\hline $\begin{array}{l}\text { Etats- } \\
\text { Unis }\end{array}$ & $\begin{array}{c}\text { Los } \\
\text { Angeles }\end{array}$ & Miami & $\begin{array}{l}\text { New } \\
\text { York }\end{array}$ & $\begin{array}{c}\text { San } \\
\text { Francisco }\end{array}$ & Europe & Amsterdam & Francfort & Londres & Paris \\
\hline $\mathrm{MCI}$ & 15 & 10 & 28 & 17 & Colt & 10 & 10 & 12 & 9 \\
\hline Level 3 & 16 & 9 & 21 & 10 & MCI & 8 & 6 & 10 & 5 \\
\hline $\mathrm{XO}$ & 10 & 12 & 21 & 11 & Level 3 & 10 & 7 & 9 & 4 \\
\hline AT\&T & 11 & 9 & 18 & 10 & Cable \&Wireless & 5 & 3 & 11 & 2 \\
\hline Qwest & 9 & 6 & 18 & 12 & KPN & 11 & 2 & 2 & 1 \\
\hline
\end{tabular}

Fig. 9/ Colocation, les métropoles choisies par les principaux opérateurs (Source : Telegeography )

Quelle que soit l'échelle géographique, la même logique domine : l'architecture du réseau est hyper centralisée et contrôlée par les plus gros opérateurs. Les flux de données traduisent la domination des Etats-Unis sur le monde mais aussi des capitales régionales sur le reste de la hiérarchie urbaine. L'observation des cartes et des graphiques montre que l'Europe paraît mieux placée que l'Asie, par rapport aux Etats-Unis. Comment expliquer ce qui est souvent énoncé comme un simple problème de « retard »?

\section{L'écosystème d'INTERNET}

Le fonctionnement d'Internet peut être comparé à un écosystème ${ }^{6}$ naturel, qui se définit comme un « ensemble d'êtres vivants et d'éléments non vivants, aux nombreuses interactions (...). C'est avant tout un système dynamique qui naît, se développe et meurt ». Ainsi le réseau Internet évolue « naturellement » en permanence, au rythme des structurations du marché, des stratégies d'acteurs, et des politiques tarifaires. On retrouve alors le modèle de M.Castells (2001) qui distingue trois dimensions dans l'espace des flux : les échanges électroniques, les nœuds d'interconnexion ou $h u b$, puis l'organisation spatiale des lieux « dominants ».

\section{Quelles sont les dynamiques du réseau ?}

Les Etats-Unis apparaissent comme « le noyau de l'Internet», concentrant les routes, les principaux noeuds, les flux, les contenus (Zook, 2002), les usagers et les principaux opérateurs. Cette première fragmentation territoriale à l'échelle du système-monde n'est pas sans effet sur le développement de l'Internet et de ses usages et a conduit jusqu'ici à un important transfert de valeur des territoires périphériques vers le centre que Cukier appelle l'«U.S.-Centricity».

\section{Un système en mutation : gratuité et logiques commerciales}

L'effondrement du système de la gratuité, qui selon certains, conférait à Internet une certaine a-spatialité, a été concomitant à son expansion. Quand l'Internet est devenu commercial (1995), la réciprocité des échanges entre les FAI a été la règle aux Etats-Unis, quelle que soit la localisation, le volume d'affaires et la nature du métier, simple loueur de bande passante ou grossiste de services internationaux.

D. Mah (2003) rappelle qu'il n'en était pas pourtant ainsi à l'ère pré-Internet (1979-1994) ${ }^{7}$. Compuserve,

\footnotetext{
${ }^{6} \underline{\mathrm{http}: / / \mathrm{www} . c a r n a v e n i r . c o m / a r t i c l e s / e c o s y s t e m e . h t m}$, consulté en janvier 2005

${ }^{7}$ «In the pre-Internet online services industry, firms like CompuServe, Prodigy and America Online offered communications and information services very similar to the services now found on the Internet.In 1994, these firms had 2.6, 1.2 and 1.5 million subscribers respectively.As late as 1994, on the eve of the commercial Internet, Microsoft launched a proprietary online service modeled on the existing services. None of these firms chose to interconnect their systems (at least not initially). They eventually joined the commercial Internet as ISPs, but this did
} 
Prodigy et America Online puis Microsoft refusaient l'interconnexion. Ils suivaient le modèle développé par les industries du télégraphe ou du téléphone qui ont alterné périodes de monopole, de compétition sans interconnexion, de collusion, puis à nouveau de monopole (1866, Western Union). C'est la politique publique de régulation qui a mis fin au monopole d'AT\&T. En fait le modèle économique de l'Internet a divergé en 1995 du modèle appliqué au téléphone. Mais si les firmes comme Compuserve, Prodigy ont migré sur Internet, c'est aussi parce que les offreurs de contenus avaient choisi ce nouveau canal, gratuit et offrant une audience plus large. Or ce choix menaçait le modèle économique des propriétaires des réseaux et les a contraint à accepter l'interconnexion.

Du point de vue des politiques publiques il est préférable que les firmes optent pour l'interconnexion et la compétition.La NSF (National Science Foundation) a souhaité tout en privatisant Internet, organiser le marché d'autant plus que la plus grande communauté d'utilisateurs provenaient des rangs du gouvernement ou des institutions universitaires. Le gouvernement fédéral subventionna alors largement l'Internet comme un «sponsor » (Katz et Shapiro, 1986). «Large buyers are natural candidates to be the network sponsors». Sa politique a réduit à la fois les coûts de connexion des FAI (subventions publiques pour les GIX) et a ainsi permis d'établir une situation de concurrence tout en assurant un maximum de connectivité, offrant ainsi aux consommateurs des effets de réseaux, une variété d'offre et des tarifs tendanciellement bas.

\section{Des interactions fortes sur les points névralgiques des réseaux}

Si les fournisseurs d'accès à Internet ne possèdent pas toujours les infrastructures qu'ils utilisent, les métiers ont tendance à s'interpénétrer afin de mieux maîtriser la chaîne de valeur.

L'organisation des questions de routage en réduisant le nombre des acteurs impliqués, donne un avantage important aux tiers one qui d'une part, vendent des circuits de transit et des services associés dans les GIX et d'autre part, ont créé des GIX privés et confidentiels pour échanger entre pairs en toute discrétion et s'assurer ainsi le contrôle de l'Internet. Les opérateurs de premier niveau tiennent à conserver leur position, comme l'évoque GTE à propos de Genuity. «Toute perte significative de marché (...) peut causer la perte de notre statut de tier one Internet backbone provider qui rendrait nos services significativement moins attractifs pour nos clients; il en résulterait une nette perte de revenus. De plus, la perte de notre statut affecterait notre capacité à maintenir notre offre de peering privé avec nos pairs. Actuellement ces relations de peering nous permettent d'échanger du trafic gratuitement avec les autres tiers one et d'éviter la congestion des GIX publics. Si nous sommes incapables de maintenir nos relations de peering actuelles, nos coûts augmenteront et nos résultats en pâtiront ${ }^{8} \gg$.

Initialement, le modèle économique faisait financer l'acheminement du trafic régional ou local des petits FAI par les propriétaires des grands backbones $(I B P)$. Aujourd'hui la règle de la réciprocité est limitée dans l'espace et s'est territorialisée. Le développement du système du transit a inversé les flux monétaires sur le réseau. Désormais, les petits FAI financent les plus grands (les tiers one) selon un système pyramidal et les territoires périphériques alimentent le cœur de l'Internet, c'est-à-dire les Etats-Unis.

Les acteurs non-américains se trouvent en situation de concurrence défavorable, d'une part par un effet de masse plus important aux Etats-Unis (plus de contenus, plus d'usagers), d'autre part en raison de l'indispensable prise en charge du coût de la connectivité intercontinentale vers au moins l'un des trois GIX Nord-Américains

\section{Un marché concentré dans les mains de quelques opérateurs}

\section{Qui sont les maîtres des réseaux ?}

Le profil des opérateurs varie en fonction de leur trajectoire historique, de la géographie de leurs réseaux et surtout de leur poids économique (part de marché). Les opérateurs de transit sont les plus importants, à la fois parce qu'ils offrent d'énormes quantités de bande passante, mais aussi parce qu'ils couvrent de larges espaces géographiques (Downes \& Greenstein, 1999 ; Cukier, 1999 ; Gorman \& Malecki, 2000). On retrouve dans cette catégorie des acteurs comme MCI/Worldcom, Qwest, PSInet et Sprint.

En 2002, MCI/Worldcom gère $30 \%$ de la bande passante des 20 plus grands backbones américains, soit bien plus que les quatre autres grands opérateurs réunis. Le réseau de MCI couvre les six continents, plus de 140 pays et plus de 2.800 villes (soit $4.500 P O P$ ). Le réseau de Qwest, raccordant près de 150 villes américaines, vise à la fois les résidentiels et les entreprises. Verio est

\footnotetext{
${ }^{8}$ Cité par G. C. Staple, in Follow the money, in Telegeography, 2000, traduit par les auteurs.
} 
implanté aux Etats-Unis, en Europe et en Asie. Verio
démarre historiquement en 1998 en offrant des services
de télécommunications à la commande publique
(administrations, établissements scolaires, etc.)
L'opérateur prend de l'importance en incorporant
plusieurs compagnies comme Iserve, Hiway,
Digitalnation, et devient l'un des plus gros hébergeurs
de sites web, avec des clients issus de plus de 170 pays.
Verio est acheté par NTT Communication en
septembre 2000, participant ainsi à devenir l'un des
fournisseurs leaders de services IP.

Ainsi, sur les 600 opérateurs recensés, les cinquante premiers contrôlent $95 \%$ du réseau. MCI/Worldcom est leader devant AT\&T (qui est pourtant l'opérateur pionnier des Etats-Unis), mais aussi devant Sprint et Genuity. En bref, les 10 plus grands opérateurs se partagent les $2 / 3$ du marché.

\begin{tabular}{|c|c|}
\hline Opérateurs & Part de Marché (\%) \\
\hline Worldcom/MCI & 27,9 \\
\hline AT \& T & 10 \\
\hline Sprint & 6,5 \\
\hline Genuity/Level 3 & 6,3 \\
\hline PSInet/Cogent & 4,1 \\
\hline Cable \& Wireless & 3,5 \\
\hline XO Communication & 2,8 \\
\hline Verio & 2,6 \\
\hline Quest & 1,5 \\
\hline Global Crossing & 1,3 \\
\hline Autres & 33,5 \\
\hline
\end{tabular}

Source : Probe Research, http://www.nwfusion.com/newsletters/isp/2001/00846039.html (11/01/2005)

\section{Fig. 10/ Part de marché (\%) des principaux opérateurs longue distance en 2000}

La chute des prix et le recul temporaire de la demande ont entraîné une vague de faillites parmi les grands opérateurs. Pourtant, on peut constater que, même au plus profond de la vague et malgré des conditions de marché très défavorables, tous les réseaux ont été maintenus en activité soit par les créditeurs soit par une entreprise rivale. $\mathrm{Si}$, malgré de nombreuses et spectaculaires faillites, on observe peu de liquidations parmi les opérateurs de réseau, on peut s'interroger sur les motivations stratégiques pour conserver la maîtrise des tuyaux. 


\section{EVOLUTION DU SECTEUR TELECOM DE 2001 à 2005 A TRAVERS LES FAILLITES, LES RACHATS, LES RESTRUCTURATIONS}

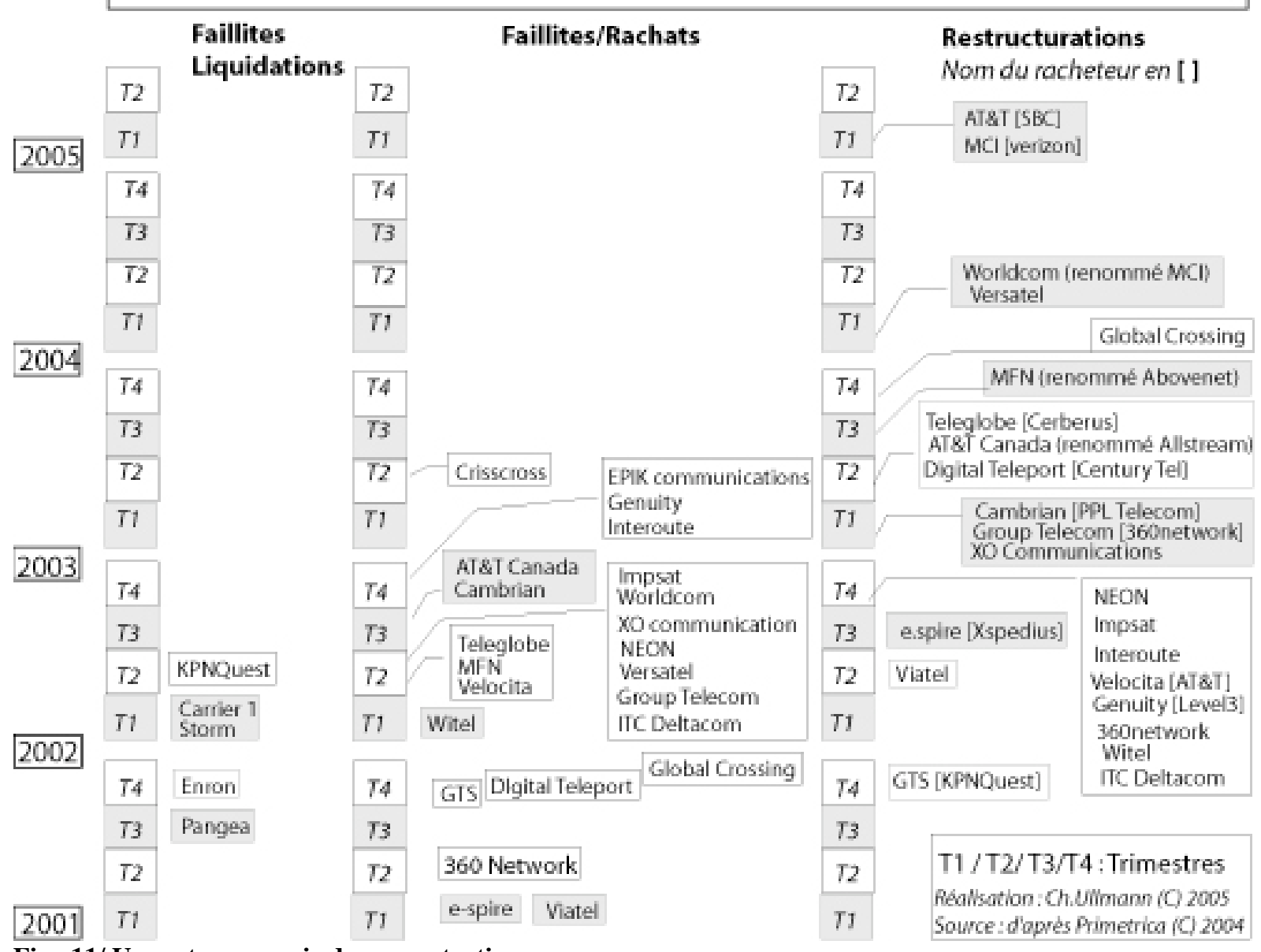

Fig. 11/ Un secteur en voie de concentration

\section{Maîtrise des réseaux et des territoires}

Les performances des réseaux peuvent se mesurer par les interactions qu'ils offrent avec les territoires (étendue de la couverture géographique, étendue de la qualité de service, liens avec les end users les plus profitables). En l'absence de métrique commune, chaque opérateur élabore sa méthodologie, destinée à le mettre en valeur vis-àvis des entreprises clientes. Quels que soient les critères choisis (points de présence, pays desservis, quantité de paquets transportés, nombre de domaines connectés), tous ont un rapport direct avec le territoire et les règles de localisation géographique (distance, accès, temps de desserte). Les indicateurs plus souvent utilisés sont de deux types : l'un basé sur la performance du réseau, l'autre sur la taille.

Tout d'abord, la redondance des points indique une qualité de service égale, garantie par l'opérateur, mais seulement sur son propre réseau. Equant, présent dans 145 pays, ou encore $\mathrm{MCI}$ /worldcom qui possède 4.500 points de présence répartis sur tous les continents, jouissent d'un avantage décisif.

De plus, la quantité de paquets transportés permet des économies d'échelles. S'il y a plus de trafic sur le réseau, paradoxalement le trafic va plus vite. $\mathrm{MCI} /$ Worldcom affirme être connecté au plus grand nombre de noms de domaines et de FAI dans le monde, ce qui influence la rapidité de la circulation sur son réseau.

La qualité de service offerte par les opérateurs (temps de latence, disponibilité, perte de paquets minimale) suit une règle géographique simple : plus on est au cœur de l'Internet, plus la qualité est assurée. Les pertes de paquets sont plus rares au cœur de l'Internet que dans la périphérie du réseau (dernier kilomètre). Le temps de latence varie selon les territoires. En 2003, il variait de 45 à 65 millisecondes, selon les opérateurs en Amérique du Nord mais pouvaient atteindre le double ou le triple sur les routes transocéaniques.

\section{Maîtriser les réseaux, une stratégie dominante?}

La croyance collective des années de la «bulle » prétendait que l'essentiel de la valorisation reposait sur les contenus et que leur contrôle était au cœur de la « révolution numérique ». I. Ramonet (2004) rappelle d'ailleurs que «d'un bout à l'autre de la planète, les seigneurs de cette guerre des réseaux sont les mêmes, des conglomérats géants devenus les nouveaux maîtres du monde (...). Sont concernées par cette guerre les firmes 
qui produisent des contenus (...) et les entreprises de télécommunications et d'informatique qui les élaborent, les transportent, les traitent, les cryptent et les décryptent. La manne dont veulent s'emparer les nouveaux prédateurs est le flux sans cesse croissant de données : conversations, messages, textes, images, musiques, films, émissions, spectacles, sport, informations, bourse».

L'hypothèse doit toutefois être renversée, car il semble que la stratégie des acteurs s'est toujours concentrée sur le contrôle des infrastructures à grande vitesse. Ce contrôle donne une position privilégiée dans le choix de la répartition de la rente. L'industrie musicale américaine ne s'y trompe pas. La RIAA, Recording Industry Association of America, poursuit depuis 2002 les grands IBP (AT\&T, Cable\&Wireless, Sprint, UUNet and Advanced Internet Services) afin qu'ils bloquent depuis leurs réseaux l'accès au serveur «Listen4ever.com» localisé en Chine, qui permet des téléchargements de fichiers musicaux MP3.

Cette main mise sur les tuyaux est un phénomène qui peut aussi être observée à l'échelle des nations. En France, l'ouverture à la concurrence du marché de l'ADSL et l'accélération du processus de dégroupage, encouragées par l'Autorité de Régulation des Télécommunications 9 , ont modifié les termes de la concurrence entre les FAI, les propriétaires de réseaux et les clients ${ }^{10}$. Jusqu'alors les FAI ne souffraient pas trop de ce handicap car les opérateurs, victimes de problèmes de surcapacité avaient besoin de vendre de la fibre ; leur intérêt était donc de préserver l'équilibre des prix avec les offres des simples FAI. Désormais les fournisseurs d'accès qui ne disposent pas de leur propre réseau sont menacés de ne pas pouvoir rester compétitifs face aux opérateurs qui composent eux-mêmes leur offre de service.

La restructuration du secteur laisse émerger des opérateurs de plus en plus «intégrés», occupant tous les maillons de la chaîne de valeur, à la fois propriétaires des infrastructures, transporteurs, et fournisseurs d'accès. Nombre d'entre eux se positionnent sur le marché de détail, allant jusqu'à fournir les services directement aux abonnés (télévision, Internet à haut débit). Pour les simples FAI, l'alternative est simple : soit racheter un opérateur, soit être vendu. Le but final est bien par la maîtrise des tuyaux, de s'assurer de la maîtrise des flux et des contenus pour capturer le plus grands nombres d'abonnés.

Paradoxalement, la bataille entraîne une déconnexion entre les prix de vente aux abonnés qui baissent considérablement et la réalité économique. Le marché se comporte comme pendant la «bulle» (offres à perte et investissements publicitaires massifs), beaucoup cherchant une valorisation boursière maximale dans un environnement économique peu favorable.

\section{La domination des réseaux par les prix}

Les opérateurs européens acheminent leurs données régionales via les Etats-Unis pour des raisons d'enjeux concurrentiels et d'effets de réseaux mais aussi pour une question de coût.

Les coûts des contrats varient selon les routes, en fonction de la distance et des destinations géographiques. Pour une distance équivalente, les coûts sont nettement moins chers aux Etats-Unis qu'en Europe (Cukier); «Le "22 à Asnières" est de retour au niveau européen : pour passer de Paris à Bruxelles le chemin le plus court passe toujours par New York ou plus exactement par Pennsauken dans le New Jersey. De plus les accords d'échange entre pairs existants sur les noeuds américains permettent une gratuité du transit vers les autres pays d'Europe alors qu'avec l'Ebone européen ils doivent acquitter des droits ${ }^{11} . »$

Ainsi, l'itinéraire Bruxelles-Londres coûte en 2002 plus de deux fois plus que le trajet NewYork-Washington. Les noeuds américains permettent un transit gratuit dans le sens retour vers l'Europe ; seul se paye le demicircuit Europe/Etats-Unis. Ce mode de fonctionnement rend encore moins attractif l'investissement sur les trajets directs. Par exemple, Paris possède seulement des liens directs avec New York, Londres, Madrid, Amsterdam, Francfort et l'Italie.

\footnotetext{
9 ARCEP depuis 2005.

${ }^{10}$ Le maintien du monopole de France Télécom se serait très certainement traduit par une offre de services moin riche, moins étendue géographiquement et des prix plus élevés pour les usagers finaux. L'analyse des réactions tactiques de France Télécom chaque fois qu'il affronte une nouvelle forme de concurrence semble conforter cette hypothèse (la livebox suit la freebox, les coûts diminuent là ou la concurrence arrive, l'ADSL se déploie sur les territoires où s'expérimentent des technologies alternatives). Cf. Fautrero V., Fernandez V. \& Puel G. (2005).

${ }^{11}$ http://yolin.net/ consulté le 24/02/2005.
} 


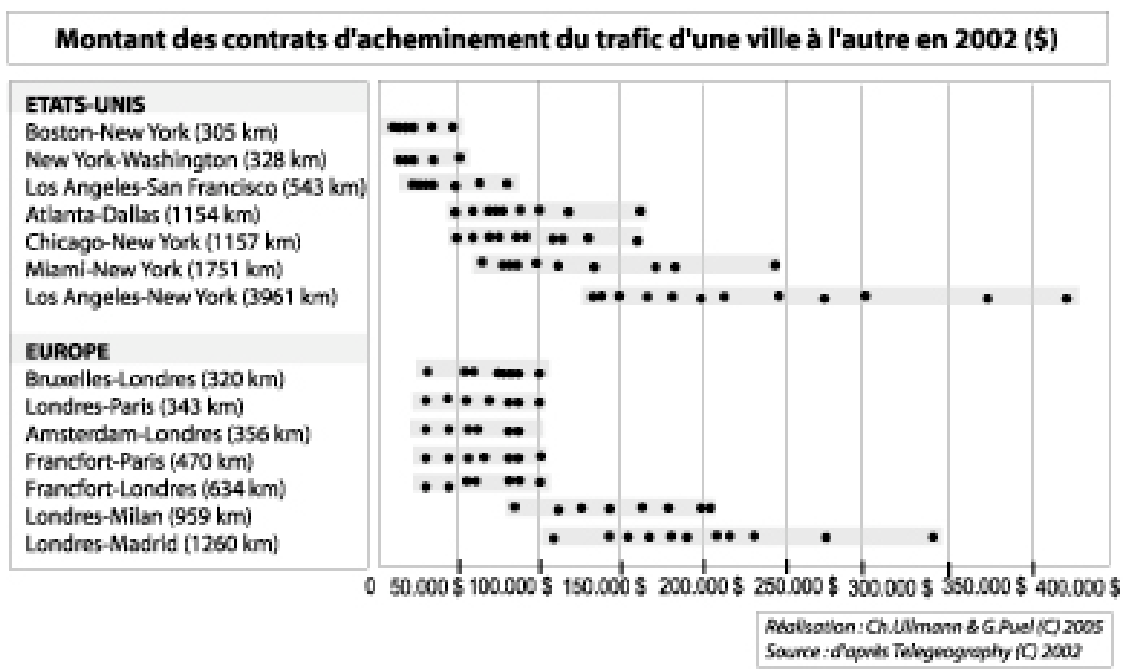

Fig. 12/ Chaque point indique la valeur d'un contrat; les prix pour une même distance peuvent varier en fonction du volume et des conditions économiques.

La situation risque de perdurer (Herring, 1999) car même sur les nœuds, il revient souvent moins cher pour deux opérateurs européens de s'interconnecter dans un GIX américain plutôt qu'en Europe. Cukier (1999) note que Telia, l'opérateur suédois, a ramené aux Etats-Unis un serveur Altavista hébergé à Stockholm car le coût de l'utilisation du réseau en Europe coûtait six fois plus cher que l'utilisation du réseau transocéanique (Telecommunications Users Group, 1999). Les mêmes logiques jouent pour l'Asie ou l'Afrique ${ }^{12}$ ou l'Amérique du Sud. Le réseau de Teleglobe acheminait, en 1999, 71,6\% du trafic singapourien vers les Etats-Unis (seulement $8,2 \%$ était à destination des Etats-Unis). Le principal GIX où se rencontrent les réseaux asiatiques est en Californie, car les opérateurs sont rétifs à une coopération en Asie.

Le territoire nord américain profite indirectement d'un avantage comparatif par rapport au reste du monde, qui connaît lui-même une situation différenciée (prix des circuits deux fois plus cher en Asie qu'en Europe). Comparativement aux Etats-Unis, l'Europe a souffert d'un manque d'opérateurs de niveau international : les fragmentations territoriales, politiques, linguistiques et monétaires ont nui à la coopération entre les grands opérateurs nationaux et à la constitution rapide de larges marchés.

\section{Conclusion}

S'il semble que l'Europe, puis l'Asie réduisent leur «retard» dans leur connectivité à l'Internet mondial (en termes de routes et de coûts), toute modification profonde de la topologie d'Internet ne peut que bouleverser les situations acquises. Malecki affirme que « les interconnexions et les paiements transforment Internet en une infrastructure hiérarchique plus apparentée aux télécommunications qu'à un Internet démocratique constitué de réseaux au même niveau les uns par rapport aux autres ». Ces droits de passage sont une des clés de l'évolution d'Internet et de ses rapports avec les territoires. Les flux monétaires suivent les mêmes routes et témoignent de cette situation inégalitaire. Les coûts de l'Internet, une condition non négligeable à son développement, témoignent de cette iniquité territoriale : ils sont moins élevés aux Etats-Unis qu'en France, moins coûteux à Paris qu'à Lyon, à Toulouse qu'à Saint-Salvy-de-la-Balme.

Lorsque les «liens» à grande distance sont aussi structurants que les liens de proximité, que deviennent les «lieux», les territoires politiques et culturels ? En fin de compte, la difficulté qui se pose à l'échelle mondiale, c'est qu'il n'existe pas d'instance de régulation ou d'organisme politique transnational qui ait l'autorité de coordonner ou contrôler les dynamiques liées aux réseaux. Ainsi, le modèle de développement de l'Internet mondial est régi par des principes commerciaux, ce qui explique la concurrence féroce entre les opérateurs.

En changeant d'échelle, les dimensions l'Internet révèlent des invariants mais aussi des changements dans les degrés et les modalités de ses interactions. Au titre des invariants, deux éléments se dégagent. La topologie des routes est liée à la hiérarchie urbaine (densité des usagers, des marchés, des acteurs), aux effets induits de congruence territoriale et à l'organisation cristallisée des réseaux de communication (poids des structures héritées de la téléphonie). Les choix technologiques sont liées à la géographie de l'accès aux marchés et non à des impératifs techniques supposés neutres. Les logiques économiques des acteurs reposent principalement sur la

\footnotetext{
${ }^{12}$ http://www3.wn.apc.org/africa/afrmain.htm, visité le 16/11/04.
} 
maîtrise des tuyaux, bien avant les contenus. Le choix des acteurs des télécommunications a été de déployer la plus grande capacité de bande passante sur les axes internationaux ou/et de contrôler les répartiteurs sur les derniers kilomètres.

Mais les variations d'échelles territoriales supposent des variations dans les angles d'analyse du système Internet car les stratégies des acteurs s'encastrent différemment.

- Au plan mondial, les autorités de régulation (U.I.T) sont impuissantes face aux opérateurs privés et aux Etats-Unis. Toutefois, les tier ones ne jouissent pas d'une totale liberté : les tentatives de fusion approchant une situation monopolistique ont été bloquées par le gouvernement américain et la Commission européenne.

- A la mésoéchelle, le rôle des autorités de régulation est souvent plus efficace (France) même s'il demeure variable (Algérie). Mais, il n'est pas facile pour les opérateurs de changer d'échelle : en témoigne le errements stratégiques de France Télécom sur l'échiquier international à travers sa filiale, Equant. Le montant de sa dette correspond à la contrepartie de transformation de France Télécom en multinationale.

- A l'échelle locale, les opérateurs historiques dominent quasiment sans partage même si quelques limites leur sont apportées par de rares opérateurs alternatifs, l'ombre portée des tier ones et la puissance publique $^{13}$. Cette dernière oscille souvent entre une politique de défense de son « champion national» et une politique d'aménagement du territoire. Il serait d'ailleurs intéressant d'interroger les politiques publiques de sponsoring sur les territoires et les stratégies des acteurs des télécommunications pour voir si les pratiques ne vont pas à l'encontre des buts affichés.

\section{Références}

Batty M.(1997) «Virtual geography », Futures, vol.29(4/5), pp.337-352.

Brown J.S. \& Duguid P. (2002), The social life of information, Harvard Business School Press, Boston, MA.

CAIDA, The Cooperative Association for Internet Data Analysis, provides tools and analyses promoting the engineering and maintenance of a robust, scalable global Internet infrastructure, http://www.caida.org/, consulté le $20 / 0 / 11 / 2005$.

Castells M., (2001), La galaxie Internet. Fayard, 366 p.

Cukier, K. N., (1999), "Bandwidth colonialism? The implications of Internet infrastructure on international ecommerce". INET' '99, San Jose, CA.

DodgeM. \& Kitchin R, (2001), An atlas of cybespace, Addison-Wesley, London.

Downes T. A. \& Greenstein S., (1999), "Do Commercial ISPs Provide UniversalAccess"?, in Competition, Regulation \& Convergence 195, Sharon E. Gillet \& Ingo Vogelsang eds.

Dufeal M. (2004), Les sites web marqueurs et vecteurs de dynamiques spatiales et économiques dans l'espace méditerranéen français, Thèse de géographie sous la direction de Loïc Grasland

Dupuy G., (2002), Internet : géographie d'un réseau. Paris : Ellipses, 160 p. Coll. « Carrefours».

Dupuy G., (2005), «Internet : une approche géographique à l'échelle mondiale », Flux N 58, pp. 5-19

Herring R., (1999), “All roads lead to the states", www.redherring.com/mag/issue63/news-telecom.html, visité le $16 / 11 / 04$.

Fautrero V., Fernandez V. \& Puel G. (2006). «Les technologies alternatives à l'usage : à propos d'une expérimentation «Satelite-Wifi » in TIC et dynamiques spatiales, Cordes Sur Ciel, 2005 http://candle.enstbretagne.fr/TICetEspace/Programme_workshop.htm, vérifié le 21/XI/2005 (à paraître).

Fullsack J-L, (2002), L'UIT la vieille dame des télécommunications dans la tourmente libérale, Strasbourg, 10$2002 \mathrm{http}: / /$ www.globenet.org/csdptt/

Graham S. \& Marvin S. (2001) Splintering urbanism : Networked infrastructures, Technological Mobilities and the Urban Condition. London : Routledge.

Gorman S. \& Malecki E. J (2000), The networks of the Internet : an analysis of provider networks, Telecommunications Policy 26

Grubesic T.H, O'Kelly M.E (2002), ’'Using point of presence to measure accessibility to the commercial Internet", The Professional geographer, Vol.54, pp. 259-284

Katz M. L. \& Shapiro C., (1986), "Technology Adoption in the Presence of Network Externalities", 94 J. POL. ECON. 822.

Mah D. C.H., (2003), Explaining Internet Connectivity: Voluntary Interconnection Among Commercial Internet Service Providers, J.S.D., Stanford Law School.

\footnotetext{
13 Les politiques publiques ne se limitent pas à une vision infrastructurelle de la technologie ; elles offrent aussi services et usages, comme les lieux d'accès public « accompagné », dont les études géographiques sont encore trop rares et limitées dans leur ambition par la relative fiabilité des données, G. Puel et M.Vidal, (2004).
} 
Malecki E. J.,(2002), “The economic geography of the Internet's infrastructure”, In Economic geography, vol. 78 , no. 4 , p. 399-424.

W.B. Norton (1999), «Peering Decision Tree», draft 1.9, 9p ; www.ecse.rpi.edu/Homepages/shivkuma/ teaching/sp2001/readings/norton-peering.pdf

Puel G. \& Vidal M., Les lieux d'accès à Internet en Midi-Pyrénées, Université de Toulouse, 2004.

Ramonet I., 2004, "le nouvel ordre Internet", in http://www.mondediplomatique.fr/2004/01/RAMONET/10615, vérifié le 20/11/2005.

Rutherford J., Gillespie A. \& Richardson R., (2004), "The territoriality of pan-european telecommunications backbone networks", GaWC Research Bulletin 136, 29p

www.telegeography.com: société privée, membre de Primetra.inc, offrant des données en ligne et des rapports sur le marché des télécommunications fixes et mobiles.

Staple G. C. (2000) “Follow the money. Network-to-Network Payments for Internet Telephony and Other IP Traffic Systems", Pages 54-68 in Telegeography 2001.

Wheeler D. C., O'Kelly M. E., (1999), "Network topology and city accessibility of the commercial Internet", in Professional geographer, vol. 51 , no. 3 p. 327-339.

Zook M.A. (2002), "Old Hierarchies or New Networks of Centrality? The Global Geography of the Internet Content Market" in American Behavioral Scientist entitled Mapping the Global Web, Vol 44. No. 10.

Zook M.A. (2000), " Internet metrics: Using hosts and domain counts to map the internet globally », Telecommunications Policy. Vol. 24 (6/7). 